\title{
ON A THEOREM OF J. A. GREEN
}

\author{
MICHAEL F. O'REILLY
}

(Received 25 July 1974)

Let $G$ be a finite group, $k$ a field of characteristic $p$ and $\Gamma$ the group algebra of $G$ over $k$. Let $e=\sum_{g \in G} \alpha_{g} g, \alpha_{g} \in k$, be a primitive central idempotent of $\Gamma$; let suppe $=\left\{g \in G: \alpha_{g} \neq 0\right\}$. We provide a short proof of a slightly stronger version of Theorem 5 of Green (1968).

THeOREM. Let $P$ be any p-subgroup of $G$ containing a defect group $D$ of $e$. There exist p-regular elements $x, y, z \in$ suppe such that $x y=z, D$ is a Sylow p-subgroup of $C(x), C(y), C(z)$ and $D=P \cap P^{x}=P \cap P^{y}=P \cap P^{z}$.

For $X \subset G$ let $\bar{X}=\Sigma_{g \in X} g$. For $H \leqq G$ let $A_{H}=\left\{\gamma \in \Gamma: \gamma^{h}=\gamma\right.$, all $\left.h \in H\right\}$. For $\gamma \in A_{H}$ and $H \leqq L \leqq G$ write $\gamma_{H}^{L}=\Sigma_{l} \gamma^{\prime}$ where $l$ ranges over a right transversal of $H$ in $L$; then $\gamma_{H}^{L} \in A_{L} . A_{L}$ has as basic the distinct $L$-orbit sums $\left\{g_{C_{L}(g)}^{L}: g \in G\right\}$. If $H \leqq C_{L}(x)$ and $K \leqq C_{L}(y), x, y \in G$ then

$$
x_{H}^{L} y_{K}^{L}=\sum_{b}\left(x^{b} y\right)_{H^{b} \cap K}^{L}
$$

where $b$ ranges over a $(H, K)$ double coset transversal in $L$. See Green (1968; Lemma 4f).

Let $L$ be a $p$-subgroup of $G$. If $S \leqq L$ and $g \in C(S)$ then

$$
g_{S}^{L}=\left[C_{L}(g): S\right] g_{C_{L}(g)}^{L}
$$

which is zero unless $S=C_{\mathrm{L}}(g)$. Hence if $\left(x^{b} y\right)_{H^{b} \cap K}^{L_{K}}$ is a non-zero term in (1) then $H^{b} \cap K=C_{L}\left(x^{b} y\right), H=C_{L}(x)$ and $K=C_{L}(y)$. So $x^{b}, y \in C\left(C_{L}\left(x^{b} y\right)\right)$. We deduce

(2) if $L$ is a p-subgroup and $z_{s}^{L}$ a non-zero L-orbit sum occurring as a summand in (1) then $z=x^{b} y^{c}$ for some $b, c \in L$ with $x^{b}, y^{c} \in C(S)$.

For such a term $C_{L}(z)=L$ if and only if $H=K=L$. It follows that the 
linear projection $\sigma_{L}: A_{L} \rightarrow A_{L}$ which annihilates terms $g_{C_{L}(g)}^{L}$ with $C_{L}(g)<L$ is an algebra homomorphism.

Let $P$ be a $p$-subgroup of $G, a \in G$. Let $S(a)=C_{P}(a)$.

Léma. If $a_{S(a)}^{p} \notin \operatorname{rad} A_{P}$ then $S(a)=P \cap P^{a}$.

Proof. If $S(a)<P \cap P^{a}$,

$$
\bar{P} a_{S(a)}^{P}=\left[P \cap P^{a}: S(a)\right] \overrightarrow{P a P}=0 .
$$

Thus for any $g \in G, \overline{P g P} a_{S(a)}^{P}=0$ i.e. right multiplication $\lambda\left(a_{S(a)}^{P}\right)$ by $a_{S(a)}^{P}$ annihilates an element of each indecomposable summand in the $P \times P$-module decomposition $\Gamma_{P \times P}=\bigoplus \Sigma[P g P]$; here the sum ranges over the $P \times P$-modules $[P g P]$ spanned by the double cosets $P g P$. Thus $\lambda\left(a_{S(a)}^{P}\right) \in$ rad End $_{P \times P} \Gamma$, (Jacobson (1943; page 60, Theorem 8). Since $\lambda$ embeds $A_{P}$ in $E_{n d P P} \Gamma, a_{9 a}^{P} \in A_{P} \cap$ $\lambda^{-1}\left\{\operatorname{rad} \operatorname{End}_{p \times P} \Gamma\right\} \subset \operatorname{rad} A_{P}$.

Proof OF Theorem. Writing $e$ as the sum of $P$-orbit sums, $e=\Sigma_{a} \beta_{a} a_{S(a)}^{P}$ where $\beta_{a} \in k$, each $a$ is $p$-regular, $D \geqq G$ any Sylow $p$-subgroup of $C(a)$ and $S(a)=D$ for some $a$ with $\beta_{a} \neq 0$. See Green (1968; Lemma 2d). Thus $e \sigma_{D} \neq 0$.

Let $\mathscr{K}=k e r \sigma_{D} \cap A_{P}, R=\mathscr{K}+\operatorname{rad} A_{P}$. The elements $e \bmod \mathscr{K}$ and $e \bmod R$ are non-zero idempotents. If $a_{S(a)}^{P} \notin R$ then

(i) $\quad a_{S(a)}^{P} \notin \mathscr{K}$ whence $S(a)={ }_{P} D$ and by suitable choice of $a, S(a)=D$ and

(ii) $a_{D}^{P} \in \operatorname{rad} A_{P}$ whence from the lemma $D=P \cap P^{a}$. Thus $e \equiv$ $\Sigma \beta_{a} a_{D}^{P}(\bmod R)$ where $S(a)=D=P \cap P^{a}$.

Since $e \bmod R$ is idempotent any $z_{D}^{P} \notin R$ in this summation-at least one must exist-occurs as a term in the product of some pair of terms $u_{D}^{P}, v_{D}^{P} \notin R$. By (2) $z=u^{b} v^{c}$ where $b, c \in P$ and $u^{b}, v^{c} \in C(D)$. Choose $x=u^{b}, y=v^{c}$. Then $z=x y, D \leqq C(x) \cap C(y) \cap C(z)$. Since $D \geqq G$ any Sylow $p$-subgroup of $C(x), C(y)$ or $C(z), D$ must be a Sylow $p$-subgroup of these groups. Since $x_{D}^{P}, y_{D}^{P}, z_{D}^{P} \notin R, D=P \cap P^{x}=P \cap P^{y}=P \cap P^{z}$.

\section{References}

J. A. Green (1968), 'Some remarks on defect groups', Math. Z. 107, 133-150.

N. Jacobson (1943), The theory of rings (Mathematical Survey A. M. S., 1943).

Department of Mathematics

University of Papua New Guinea

Port Moresby

Papua New Guinea. 